\title{
Thermal and Acoustic Properties of Agar Phantom Fabricated from Agar Powder
}

\author{
Ogbonna Nnamuchi \\ Email: prael2003@yahoo.com
}

\begin{abstract}
We present in this paper, step by step procedure in obtaining Agar Phantom from Agar Powder and degassed water using specified bioinstruments, therewith subsequently demonstrate by the Step - Wise Transient Method (STM), how the manufactured (TMM) was utilized in identifying the thermal, and acoustic properties of the fabricated material. TMMs are materials which mimic human body tissues, and as human body tissues are not readily easy to come by, Agar Agar has been used for fabrication of this suitable alternative to simulate the properties of human body tissues in certain medium.
\end{abstract}

Keywords: Phantom, Transducer, Agar, Ultrasound, Step - wise Transient Plane Source (STPS) Method.

DOI: $10.7176 /$ ISDE/12-1-05

Publication date: March $31^{\text {st }} 2021$

\section{Introduction}

Phantoms or imaging phantoms are highly specialized objects used in medical imaging for quality control, equipment calibration, dosimetry and education. The name is used interchangeably for each object despite their differences.

\subsection{Definition}

Two types of phantoms exist: Anthropomorphic and calibration.

\subsubsection{Anthropomorphic Phantoms}

Anthropomorphic Phantoms are made of materials with similar tissue characteristics to normal biological tissues/organisms. Due to their availability and likeliness to real patients, anthropomorphic phantoms can be used for a variety of tasks. Rather than image multiple patients or patient tissues, anthropomorphic phantoms can be used for trial and error to assess the optimal use of radiation such as in new protocols or image reconstruction techniques. In the same vein anthropomorphic Phantoms can be utilized for teaching students, and/or Staff different imaging techniques or exposure factors. Recent Biomedical Engineering advancements are improving the development of anthropomorphic phantoms to better mimic patient tissue.

\subsubsection{Calibration Phantoms}

A calibration phantom is often a cylinder, or plate with densities of already known values. They are utilized in quality control to ensure images are reconstructing the imaged phantom to the correct density values. Deviation from these values can indicate a need for imaging equipment service.

In the perspective of this work, a phantom is a representation of something abstract, ideal, or incorporeal. The following process lead to fabrication of Anthropomorphic Phantoms from Agar powder. This Phantom can be made from Agar Powder or Agar Agar Powder (In this case, non - edible) Some Agar are edible. This was noted for precautions to avoid harm due to injestion. We present the results of experiment for the manufacture and testing of the thermal and acoustic properties of this material. In this study, the Step - wise Transient Plane Source (STPS) Method was used in establishing the thermal property, while the Single Transducer method was used for the acoustic properties measurement of the fabricated Phantom. 


\section{Materials and Method}

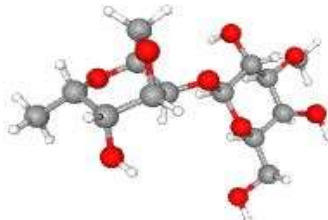

Fig. 1: Agar Chem. Structure (Pubmed)

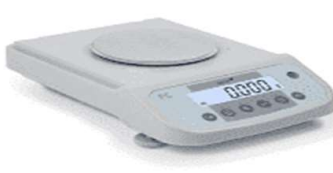

Fig. 2: Weighing Pan

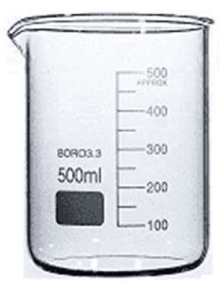

Fig. 3: Beaker

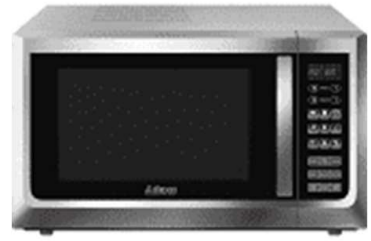

Fig.4: Microwave Ove

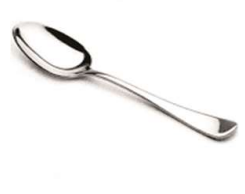

Fig. 5: Table Spoon

(a) $2 \mathrm{~g}$ of Agar Agar $\left(\mathrm{C}_{14} \mathrm{H}_{24} \mathrm{O}_{9}\right)$ powder was measured with table spoon from a plastic container of the non - edible type using the Electronic Weighing Pan (EWP).

Agar agar is a tan powder (NTP, 1992). Agar is a complex sulfated polymer of galactose units, extracted from Gelidium cartilagineum, Gracilaria confervoides. It is related to red algae. It can be used as a gel in the preparation of solid culture media for microorganisms, as a bulk laxative, in making emulsions, and as a supporting medium for immunodiffusion and immunoelectrophoresis.

(b) The $2 \mathrm{~g}$ Agar powder was poured into the cylindrical cup (Beaker) with $100 \mathrm{ml}$ degassed water. During degassing, dissolved gases are removed from the water. Oxygen and carbon dioxide are absorbed by water in certain quantities, so they need to be removed to eliminate gas bubbles which might have negative impact on the results of using the Phantom produced for Ultrasound purposes. The technical removal of troublesome, gaseous contents is generally referred to as "degassing" [6]. The mixture was then boiled at about $100^{\circ} \mathrm{C}$.

(c) The mixed Agar was stirred and microwaved 3 minutes in a Microwave oven.

(d) In each period of microwaving, the preparation was checked by viewing through the lid of the Microwave to ascertain when it is vaporizing, so as to stop it to a avoid spillage inside the equipment which is not advisable for such electrical machine.

(e) To avoid Agar suspension in the solution, we removed suspension by stirring to clear gas bubbles.

(f) The solution was for a number of times microwaved and stirred to further remove gas bubbles.

(g) The preparation was checked to ascertain when the volume of the solution had diminished in the container (beaker) as this could affect the quality of phantom produced.

(h) Degassed water level was topped up to ensure maintenance of $100 \mathrm{ml}$, while ensuring there were no gas bubbles in the solution.

(i) Then, we put the preparation beaker containing Agar solution with no gas bubbles on a magnetic stirrer and set the stirring knob to above stirring speed of 2 until the temperature is a little above $95^{\circ} \mathrm{C}$.

(j) Still checked and removed any more gas bubbles, and pour formation into a meter mold.

(k) The container of the wet preparation was labelled and dated and put in the refrigerator for the agar Phantom to set overnight.

N/B: Hot Plate Method can be used in Agar Phantom fabrication, but Microwave approach was adopted to speed up the process.

\section{Measurement of Thermal Property of Agar Phantom}

\section{(a) Thermal Experiment Set-up}

The detailed set up for the thermal property measurement using the fabricated agar phantom is shown in the diagram below (Fig. 6): 


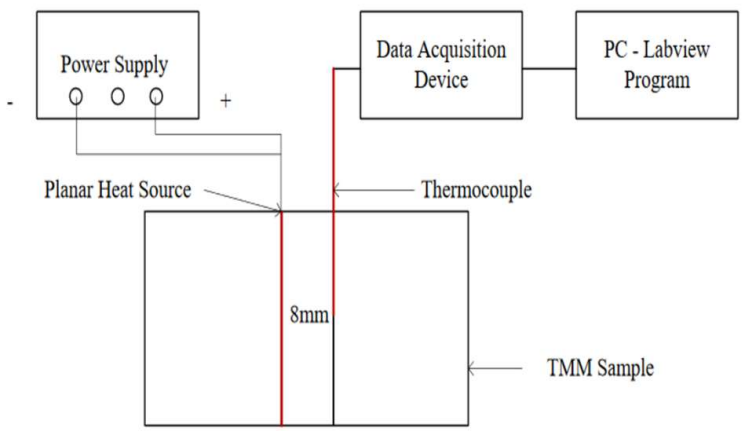

Fig 6: Pictogram illustrating experimental set up for Thermal Property Measurement

Following the experimental set up above:

1. The thermocouple' sensor was aligned into the phantom at an angle of $90^{\circ}$ to the surface. The Phantom rests and at a height of $1 \mathrm{~cm}$ from the top surface of the Phantom. The LabView application was allowed to run for no more than 600 seconds.
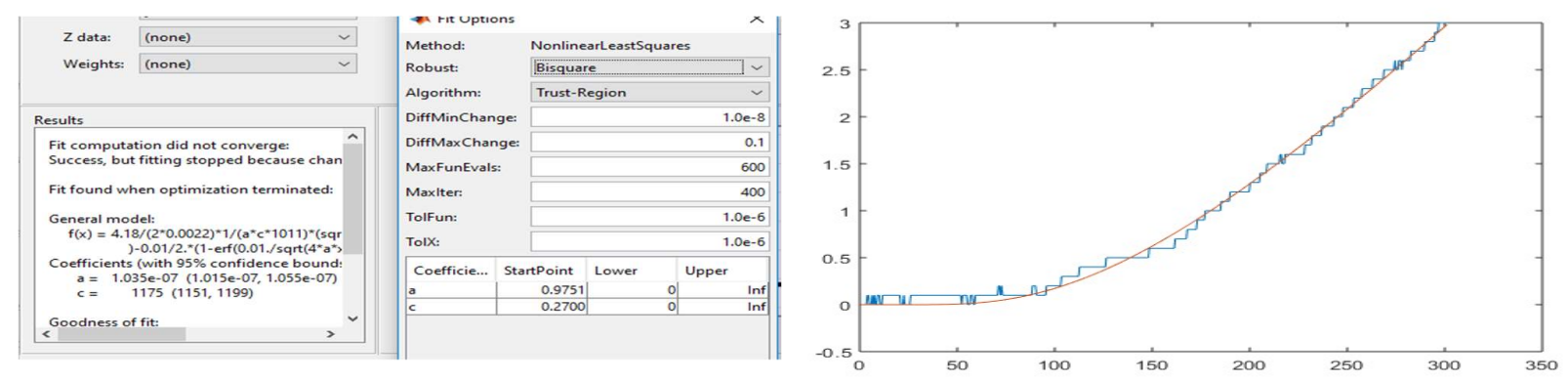

Fig. 7: $D=1 \mathrm{~cm}$ (Curve Fitting for Phantom resting $1 \mathrm{~cm}$

2. Alignment of the thermocouple's sensor into the phantom at an angle of $90^{\circ}$ to the surface of the phantom and resting at a height of $2 \mathrm{~cm}$ from the top. A Labview application of no more than 600 seconds was maintained. Precisely maintained for 300 seconds in the first Labview application, and for 470 seconds in the second. The results obtained by the execution of the LabView was extracted and saved to an external excel file containing the timings and the amplitudes at each of the time intervals. Next was to show the work done on the two format files of the results of no more than 600 seconds of data acquisition from both experiment set up.
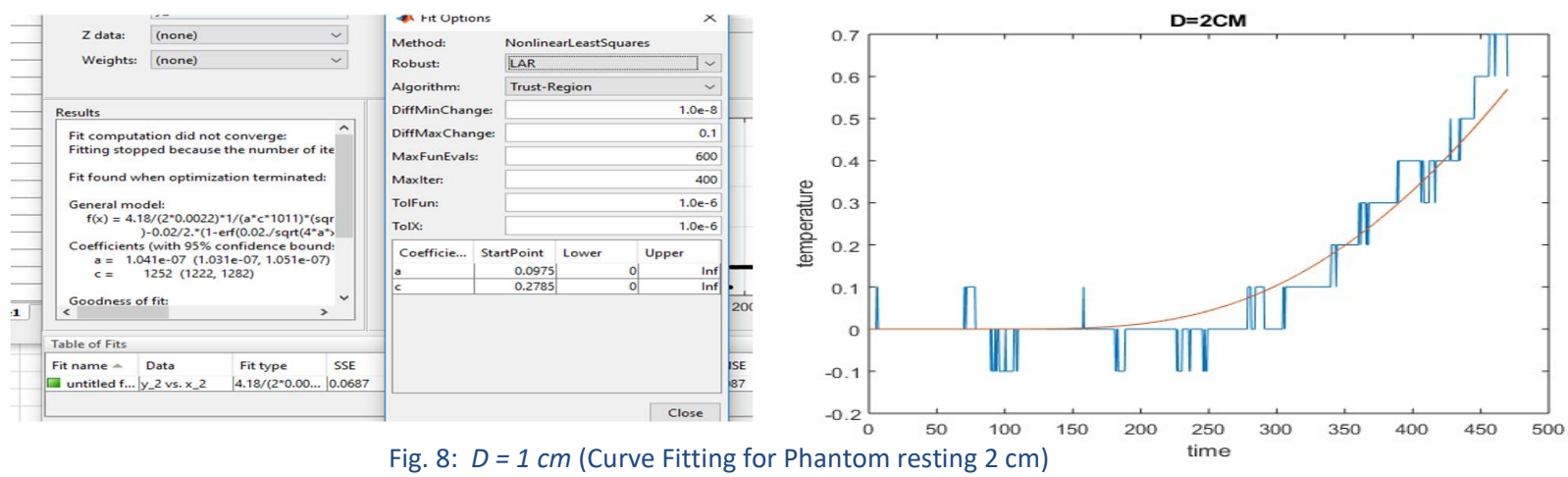

Fig. 8: $D=1 \mathrm{~cm}$ (Curve Fitting for Phantom resting $2 \mathrm{~cm}$ ) 


\section{(b) Measurement of Thermal Property of Agar based Phantom}

The Step - wise transient Plane Source (TPS) Method was used for the measurement of the thermal properties of the tissue mimicking phantom. The step - wise transient method is a method used for the measurement of the thermal diffusivity and thermal conductivity of a material, in this case the material is the phantom [3]. To determine thermal properties of materials, it is sufficient to measure the temperature as a function of time by only one thermocouple [1]. See figure 9 for a diagram showing set up of the experiment.

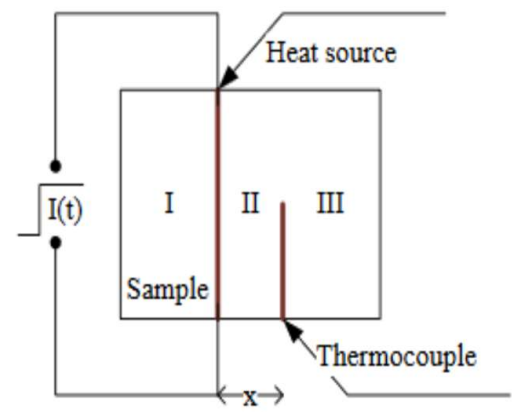

Fig 9: Set up showing step-wise transient method

Following are the results obtained from the raw data exported into Excel worksheet before simulation in MathLab, using curve fitting to obtain the results of the thermophysical parameters $\alpha=a$, and c: So we obtain $k$, using $k=\alpha . c . \rho$

\section{Detailed set up of Acoustic Property Measurement}

\section{$b_{1}$. Single Transducer Method (Without Phantom)}

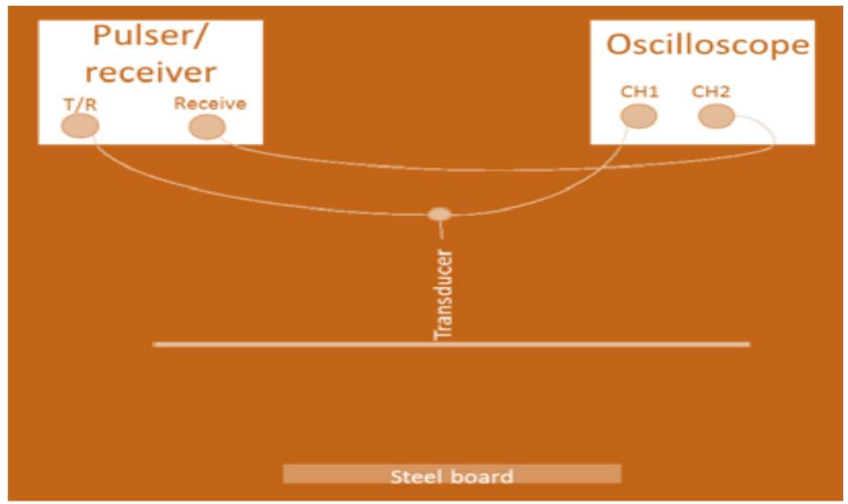

Fig. 10: Pictogram of the setup of the Single Transducer Method (without phantom)

In the single transducer method, the transducer serves the dual place as the ultrasound generator and the ultrasound receiver. In this case measurements were obtained as follows: Distance from the steel board to the transducer at $8.5 \mathrm{~cm}$ in $114.5 \mu \mathrm{s}$. The density of water is $1000 \mathrm{~kg} / \mathrm{m}^{3}$. It is natural to follow the density of Phantom is $1000 \mathrm{~kg} / \mathrm{m}^{3}$ as well. The aim of this set up is to enable us quickly obtain the ultrasound' velocity in the water, given by:

$$
V w=\frac{2 D}{d t}
$$

Where $d t$ (in seconds) corresponds to the time needed for the reception by the transducer of the first echo, where $D$ is the distance between the steel board and the transducer, measured in meters $(m)$. From the experimental results 
obtained we have $114.5 \mu \mathrm{s}$ as the time $d t$ which corresponds to the time needed for the reception by the transducer of the first echo and $D=8.5 \mathrm{~cm}$ when measured with a meter rule.

$$
\text { This yields } V w=\frac{2 \times 8.5}{d t}=\frac{17 \mathrm{~cm}}{114.5 \mu \mathrm{s}}=\frac{17000000}{11450}=1484.72 \mathrm{~m} / \mathrm{s}
$$

This looks nice as it corresponds to the standard Velocity of Ultrasound in water which is at $1480 \mathrm{~m} / \mathrm{s}$ [4]

$b_{2}$ Single Transducer Method (With Phantom) - The setup is as shown in Fig. 11 below:

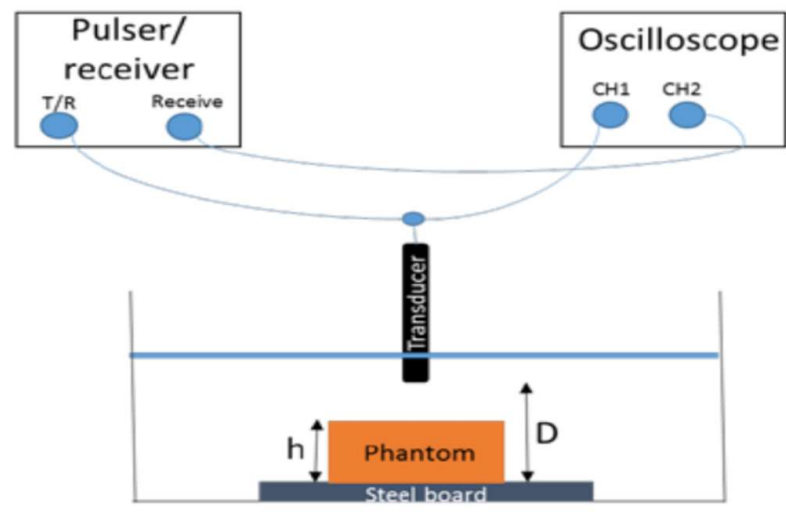

Fig. 11: showing the setup of the Single Transducer Method (With Phantom)

In the steps of $\boldsymbol{b}_{2}$, the Phantom was added between the two transducers with a thickness of $h$, shown by Figure 11 . We obtained $h=4.5 \mathrm{~cm}$. First and second echoes were obtained at $52.2 \mu \mathrm{s}$ and $114.4 \mu \mathrm{s}$, representing the times for the signals reflected by the top of the phantom and the time for the corresponding signal reflected by the border between the steel board and the bottom of the phantom. Required is the velocity of Ultrasound speed into a Phantom, designated $V_{p}$. The equation:

$$
\frac{2(D-h)}{V_{w}}+\frac{2 h}{V_{p}}=d t^{\prime}
$$

was used, where the parameters are as defined below:

$D(m)$ is distance between the transducer and the steel Board, $m$, meters

$h(m)$, is phantom thickness, $m$, meter

$V_{\mathrm{w}}(\mathrm{m} / \mathrm{s})$, velocity of Ultrasound in the water

$V_{\mathrm{P}}(\mathrm{m} / \mathrm{s})$, velocity of ultrasound in the Phantom

$d t^{\prime}(\mathrm{s})$, time between the original signal delivered by the transducer and the second echo.

Simply put, $V p=\frac{2 h}{d t^{\prime}}=\frac{2^{\wedge} 4.5 c}{114.40-52.20}=\frac{9 \mathrm{~cm}}{62.2 \mu \mathrm{s}}=\frac{0.09}{0.0000622}$

$V p$ represents the Acoustic Velocity of the ultrasound while in the phantom.

$$
V_{\mathrm{p}}=1446.94 \mathrm{~m} / \mathrm{s}
$$

Finally, we compute the Acoustic Attenuation Coefficient using the rearranged formula:

$$
\alpha=\frac{20}{h} \log \left(\frac{4 z_{p} z_{w}}{z_{p+} z_{w}} X \frac{A^{\prime}}{A^{\prime \prime}}\right)
$$


$Z=\rho^{*} V$, the acoustic impedance (in pas. $/ \mathrm{m}^{3}$ ) with $\rho$ in $\mathrm{k} / \mathrm{m}^{3}$ and $V$ in $\mathrm{m} / \mathrm{s} . A^{\prime}$ and $A^{\prime \prime}$ are the first and second echo amplitude $(V)$, while $h$ is the thickness of the Phantom. Further, we obtain the following properties:

$Z w=1000 \times V_{\mathrm{w}}=148720$ pas. $/ \mathrm{m}^{3} ; Z_{\mathrm{p}}=1000 \times V_{\mathrm{p}}=1000 \times 1446.94=1446940$ pas.$/ \mathrm{m}^{3}$

$A^{\prime}=18.5$ and $A^{\prime \prime}=3.25$ and $h$ is already known to be $4.5 \mathrm{~cm}=0.045 \mathrm{~m}$

Substituting the parameters, and calculating for $\alpha$ with the formula above, we actually obtained the value $\alpha=2884.42 \mathrm{~dB} / m=28.84 \mathrm{dBcm}^{-1}$ (bone has a range of high attenuation category $\geq 8.7 \mathrm{dBcm}{ }^{-1}$ at $f=1 \mathrm{MHZ}$ [2]).

\section{Conclusion}

This work has illustrated how Tissue Mimicking Phantom (TMMP) can be fabricated from Agar powder. The experimental process for determining the Thermal and Acoustic properties of the material manufactured has been demonstrated. Since the 1960s, tissue - mimicking materials (TMMs) have been utilized for the preparation and characterization of ultrasound imaging Wall-less flow phantoms are as well utilized to examine the performance of ultrasound device for practicing sonography. The achievement of equivalent TMM is necessary to process for a quality monitor of Doppler ultrasound diagnostic instrument. It is essential that chemical items utilized in fabricating TMMs are prepared in a planned method to be nearly equal to the acoustical properties of real tissue with attenuation and speed of sound respectively. This objective has been achieved in this work [4].

\section{Acknowledgment}

The author is grateful for sponsorship from TETfund Nigeria through Chukwuemeka Odumegwu Ojukwu University uli, Anambra State. This has enabled me to participate in this study and more. I acknowledge the team of Laboratory staff at the Bioinstrumentation Laboratory of the University of Dundee, where the fabrication practical, and machine work was done. I am very grateful for the equipment and material support I received from the group, and from the Biomedical Engineering team.

\section{References}

[1] Gao, J., You, J., Cochran, S., Corner, G., \& Huang, Z. (2012). Simultaneous measurements of thermo physical properties of egg white phantoms for HIFU by using the step-wise transient plane source technique.

[2] https://lib.dr.iastate.edu/cgi/viewcontent.cgi?article $=18317 \&$ context $=$ rtd, pp.41, accessed again, $21 / 2 / 2021$

[3] https://courses.washington.edu/bioen508/Lecture6 - US.pdf

[4] https://radiopaedia.org/articles/phantom

[5] https://search.molinstincts.com/search/searchTextList.ce

[6] https://web.stanford.edu/class/rad225/subdir/Class3.pdf

[7] https://www.hydrogroup.biz/fileadmin/redakteur/pdf/product-manual/processes-for-degassing-r4i1en.pdf, accessed, 21/2/2021

[8] https://www.molinstincts.com/formula/Agar-cfml - CT1096482233.html, accessed 21/2/2021

[9] https://www.sciencedirect.com/science/article/pii/S0022407306000124

[10] Oglat AA, Matjafri et al (2018). A New Scatter Particle and Mixture Fluid for Preparing Blood Mimicking Fluid for Wall - Less Flow Phantom., J Med Ultrasound. 26(3):134-142

[11] Peter K., Svotozar M. (2014). Using the Transient Plane Source Method for Measuring Thermal Parameters of Electroceramics: World Academy of Science, Engineering and Technology International Journal of Mechanical and Mechatronics Engineering Vol:8, No 5 